\title{
WHEN TOOTHACHE RESULTS IN ASTHMA DIAGNOSIS
}

\author{
MYRIAM RAHIMO, JOANNA JERZYŃSKA, AGNIESZKA BRZOZOWSKA, and DANIELA PODLECKA
}

\author{
Medical University of Lodz, Łódź, Poland \\ Copernicus Memorial Hospital, Korczak Paediatric Center, Department of Pediatrics and Allergy
}

\begin{abstract}
Local anesthetics (LA) have been used for a wide variety of procedures over the years due to their analgesic effect. These drugs have been seen to cause adverse events in the pediatric population, but an actual allergy must be in question. A case of an apparent hypersensitivity reaction to LA used in the setting of dental procedures in a 14.5-year-old girl with a forgotten history of asthma was reported and medical documentation review was performed. After treatment with LA during several dental procedures, the patient presented the shortness of breath, malaise and fainting, which then resolved spontaneously. After proper history taking, and skin and provocation tests, the patient was diagnosed with bronchial asthma and emotional sensitivity. The patient's recommendation included using an antihistamine and controlling her asthma before the use of LA, and administering the drug in a supine position. It is essential to consider all possible etiologies of an adverse event after using drugs in the pediatric population and to perform proper testing before making the diagnosis of a drug allergy. Int J Occup Med Environ Health. 2022;35(1):107-10
\end{abstract}

Key words:

children, asthma, anaphylaxis, drug allergy, local anesthetic agents, dyspnea

\section{INTRODUCTION}

According to the statement by the World Health Organization, an adverse drug reaction is defined as any harmful, unintended and undesired effect of a drug that occurs at doses used for treatment, prevention or diagnoses [1]. Most of these reactions are classified as type A reactions which, by definition, are predictable, common, dose-dependent and caused by known pharmacological actions of the drug, drug toxicity and side effects $[1,2]$. Allergic reactions are qualified as type B reactions independent of the drug dose, affecting a small population, suggesting that individual patient host factors are important [2]. Drug hypersensitivity reactions are classified as immediate and non-immediate reactions. Usually immediate reactions occur within $1 \mathrm{~h}$ after drug administration and they are thought to be caused by direct mast cell activation or IgE mediated hypersensitivity [3]. Non-immediate reactions occur after $1 \mathrm{~h}$ after drug administration and are usually due to antigen-specific IgG production, complement activation or T-cell mediated response [2-4].

Many children previously diagnosed with a drug allergy are proven to be tolerant to the specific medication when challenged [5]. Common drugs with apparent hypersensitivity reactions in many children include $\beta$-lactam antibiotics, non-steroidal anti-inflammatory drugs and local anesthetics (LA). The latter have been widely used for years to prevent pain in dental, surgical, or ophthalmic procedures. They are classified as amides and esters depending on their lipophilic aromatic ring connection to a hydrophilic group by a linking chain [6]. These anesthetics can be used topically or subcutaneously to provide temporary analgesia by affecting the neural voltage-gated sodium channels [7-9].

Received: December 10, 2020. Accepted: May 26, 2021.

Corresponding author: Joanna Jerzyńska, Medical University of Lodz, Copernicus Memorial Hospital, Korczak Paediatric Center, Department of Pediatrics and Allergy, Piłsudskiego 71, 90-329 Łódź, Poland (e-mail: alergol@kopernik.lodz.pl). 
Amide anesthetics are more preferred than esters due to less unfavorable outcomes. Adverse effects with local anesthetics have been widely reported, but only $<1 \%$ of all reported reactions were true IgE mediated allergic reactions [8]. An adverse drug reaction is defined as a response to a drug, which is harmful and unintended [5].

Here the authors presented a report on a pediatric patient with an adverse drug reaction similar in presentation to hypersensitivity following the LA use during a dental procedure. They discussed the case as well as reported the diagnostic and treatment strategies which can be implemented.

\section{MATERIAL AND METHODS}

Written consent regarding the case report was obtained from parents. The patient's anonymity was preserved. A 14.5-year-old girl was admitted to the ward on an elective basis to diagnose allergies to LA. The first episode of an adverse reaction after LA occurred about 5 years before, during a dental procedure in which lignocainum was used as LA. The patient fainted without the loss of consciousness. As reported by the girl, there was a slight shortness of breath, following which the symptoms resolved spontaneously after changing the position. After about a year, a similar event took place when applying an articulated swab to the gum. The patient reported about 2-minute-long shortness of breath and general malaise, which also resolved spontaneously. The last episode, in November 2019, involved administering articaine at the Institute of Dentistry, following which the girl experienced visual impairment and heavy breathing about 5 min after administration, these symptoms once again resolving spontaneously. At that time, no further dental procedures were performed.

On admission to the hospital for expanded diagnostics of drug allergy, medical history was reviewed since the patient's birth. After several attempts to get a detailed medical history, the patient's mother recalled her daugh- ter being diagnosed with allergic rhinitis and probable bronchial asthma when she lived in Ukraine at the age of 5 . The patient was then under the periodic control of a pulmonology out-patient clinic. There was no regular treatment of allergy beside periodic nasal steroids and salbutamol on an emergency basis. The child was under pulmonological control up to the age of 7 , and then she was released from regular control due to the remission of allergic symptoms.

\section{RESULTS}

At the time of admission, the child was in a good general condition and the physical examination did not reveal any deviations from the normal state. The results of diagnostic tests showed an elevated total IgE level (849 IU/ml), specific IgE for house dust mites in classes 5 and 6 , and for grass pollens in class 3. Pulmonary function tests were performed during the hospitalization. In the bronchial reversibility test, $\mathrm{FEV}_{1}$ improved by $11 \%$ with an improvement in the study curve. In plethysmography, a reduced airway resistance by more than $100 \%$ was observed. Nitric oxide in exhaled air was slightly increased to $27.9 \mathrm{ppb}$.

While setting up the intravenous route, the girl reported feeling unwell and fainted. The child's well-being improved after changing to a supine position.

Skin prick tests and an intradermal test were performed with articaine and mepivacaine, both yielding negative results. Due to the patient's and her mother's high anxiety before administering articaine, a provocation test with an alternative drug (mepivacaine) was proposed in order to safely perform dental procedures. Another provocation test with articaine is being planned in the future. Subsequently, intradermal tests with mepivacaine were performed, and were also negative. Next, a provocation test with mepivacaine was performed to obtain the doses necessary for LA without adverse effects. During the provocation test and the day after, no adverse events were noted. 
Based on the history, the physical examination, and the results of diagnostic tests, bronchial asthma and emotional hypersensitivity were diagnosed. Immediate allergy to LA has not been confirmed. The girl was discharged home in a good general condition. Asthma treatment with inhaled steroids and antihistamines was ordered. Due to the girl's sensitivity to stress (prick, blood collection, dental procedures), the patient and her parents were also advised to perform the procedures mentioned above in the supine position after the oral administration, about $1 \mathrm{~h}$ before the procedure, of $25 \mathrm{mg}$ of hydroxyzine. In the case of dyspnea and malaise during procedures, the Trendelenberg position should be used altogether with an additional dose of budesonide/formoterol.

\section{DISCUSSION}

Drug reactions have been widely reported in the pediatric population, but only a small part of them were actually evaluated using tests such as drug hypersensitivities [4,5]. Drug hypersensitivity is a type of an adverse drug reaction that is sometimes difficult to distinguish from an allergic reaction [5].

Due to the wide range of reactions that can mimic LA allergy, and the rarity of a true IgE mediated LA allergy, other causes of reactions must be explored. A majority of the reactions were due to psychomotor responses, allergy to concomitantly administered agents, response to procedural trauma and delayed hypersensitivity reactions [8]. The most common adverse reaction during LA use in the setting of dental procedures is mainly psychogenic [10]. Psychomotor responses can include vasovagal syncope which is triggered by intense emotional stress, pain, fear and fatigue, and are preceded by pallor, lightheadedness, diaphoresis and nausea. Other possible psychomotor responses can include hyperventilation/panic attack and sympathetic stimulation [11]. All the possible triggers of psychomotor responses should be considered in those patients who present with adverse reactions following LA use due to their similarity in the presentation with hypersensitivity reactions. If the administration of LA accidentally gets injected into the vascular circulation, a toxic systemic reaction may occur [12].

Other common reactions to local anesthetics that must be considered include reactions to concomitantly administered agents (latex, antibiotics, additives, and preservatives). Parabens are preservatives used in amide and ester LA. Exposure to paraben containing products can sensitize an individual susceptible to LA, causing an adverse reaction to LA [8].

Clinical history taking is critical in determining the correct diagnosis and the classification of drug hypersensitivity in children [9]. A confirmation of allergy can be done using skin prick tests, intradermal tests, and subcutaneous challenge tests, with skin prick tests being the most common, and subcutaneous challenge being the most definitive. These tests are not only necessary in determining the presence of a drug allergy. They are also used in deciding safe alternatives and appropriate drug doses specific to the patient that will not cause an adverse event.

The patient was treated with mepivacaine as a safe drug, and the appropriate dose of the drug was established for use in the event of the need for dental procedures in this patient. Importantly, thanks to the diagnostics carried out, the patient underwent several dental procedures with the use of LA (mepivacaine) without any side effects. Her reactions to LA were likely psychogenic. To come to the conclusion of a psychomotor etiology of her reaction, several tests were performed, including the skin prick test, the intradermal test, and the provocation test to rule out other causes. All of these tests came back negative for immediate LA allergy. The patient's reaction was due to the intense emotional stress related to dental procedures, uncontrolled asthma, and the spontaneous resolution of symptoms following cessation of the procedure. It is also essential to recognize bronchial asthma in this patient and its relationship to emotional triggers leading to 
the shortness of breath. The final recommendations given to the patient were related to controlling asthma and catering to the patient's emotional stress.

\section{CONCLUSIONS}

Patients presenting with a hypersensitivity reaction to LA should be sent for further investigation before the diagnosis is formulated. It is important to consider the psychogenic origin of the reaction, especially in the pediatric population. Recommendations to the patients should be based on the results of the diagnostic tests, including relieving fear in the child and administrating LA in a cold room, in a supine position, and with the prior use of antihistamines such as hydroxyzine. In case the patient has other coexisting conditions, all steps required to establish the proper disease control should be considered.

\section{REFERENCES}

1. International drug monitoring: the role of the hospital. World Health Organ Tech Rep Ser. 1969;425:5-24.

2. Demoly P, Adkinson NF, Brockow K, Castells M, Chiriac AM, Greenberger PA, et al. International Consensus on drug allergy. Allergy. 2014;69:420-37.

3. Caffarelli C, Franceschini F, Caimmi D, Mori F, Diaferio L, Di Mauro D, et al. SIAIP position paper: provocation challenge to antibiotics and non-steroidal anti-inflammatory drugs in children. Ital J Pediatr. 2018;44(1):147.

4. Park JS, Suh DI. Drug Allergy in Children: What Should We Know. Clin Exp Pediatr. 2020;63(6):203-10, https://doi. org/10.3345/kjp.2019.00675.
5. Piccorossi A, Liccioli G, Barni S, Sarti L, Giovannini M, Verrotti A, et al. Epidemiology and drug allergy results in children investigated in allergy unit of a tertiary-care paediatric hospital setting. Ital J Pediatr. 2020;46(1):5, https://doi. org/10.1186/s13052-019-0753-4.

6. McLure HA, Rubin AP. Review of local anaesthetic agents. Minerva Anestesiol. 2005;71:59-74.

7. Peroni D, Pasini M, Iurato C, Cappelli S, Giuca G, Giuca MR. Allergic manifestations to local anaesthetic agents for dental anaesthesia in children: a review and proposal of a new algorithm. Eur J Paediatr Dent. 2019;20(1):48-52, https:// doi.org/10.23804/ejpd.2019.20.01.10.

8. Bhole MV, Manson AL, Seneviratne SL, Misbah SA. IgE-mediated allergy to local anaesthetics: separating fact from perception: a UK perspective. Br J Anaesth. 2012;108(6): 903-11, https://doi.org/10.1093/bja/aes162.

9. Campanella V, Libonati A, Nardi R, Angotti V, Gallusi G, Montemurro E, et al. Single tooth anesthesia versus conventional anaesthesia: a cross-over study. Clin Oral Investig. 2018;22(9):3205-13, https://doi.org/10.1007/s00784-0182413-2.

10. Grzanka A, Wasilewska I, Śliwczyńska M, Misiołek H. Hypersensitivity lo local anesthetics. Anaesthesiol Intensive Ther. 2016;48(2):128-34.

11. Harboe T, Guttormsen AB, Aarebrot S, Dybendal T, Irgens A, Florvaag E. Suspected allergy to local anaesthetics: follow-up in 135 cases. Acta Anaesthesiol Scand. 2010;54(5): 536-42, https://doi.org/10.1111/j.1399-6576.2009.02193.x.

12. Yujin R, Jewoo L, Jiyoung R. Hypersensitivity to Local Anesthetics: A Case Report. J Korean Acad Pediatr Dent. 2017; 44(3):365-9.

This work is available in Open Access model and licensed under a Creative Commons Attribution-NonCommercial 3.0 Poland License - http://creativecommons.org/ licenses/by-nc/3.0/pl/deed.en. 